\title{
Le sérum de cobaye citraté peut-il être utilisé comme complément de l'épreuve de lyse
}

\author{
par O. MORIN et C. VERMEIL \\ (Collaboration technique : F. PARoIs) \\ Laboratoire de Parasitologie et Pathologie exotique ( $\mathrm{P}^{\mathrm{r}}$ C. VermeIL) \\ Université d'Enseignement, de Recherche de Médecine \\ et de Techniques médicales, $F 44000$ Nantes
}

\begin{abstract}
Résumé
52 sérums de cobayes âgés de 3 semaines ont été étudiés comme source possible de «facteur accessoire» de l'épreuve de lyse (=dye-test) en toxoplasmose. $25 \%$ des cobayes ont donné des résultats intéressants lorsqu'ils sont dilués en solution d'Alsever au $1 / 10^{\circ}$. La même solution d'Alsever diluée au $1 / 5^{\circ}$ permet d'améliorer les premiers résultats en augmentant le pouvoir activateur des sérums. Sans l'addition de cette solution, le pouvoir lytique non spécifique de tous les sérums titrés engendrait une lyse totale tes toxoplasmes. Il semble donc que la solution d'Alsever agit d'une part en augmentant le pouvoir activateur des sérums de cobaye, d'autre part, en diminuant la lyse non spécifique des sérums de cobaye permettant ainsi l'utilisation des sérums de ces rongeurs comme complément de l'épreuve de lyse en toxoplasmose.
\end{abstract}

\section{Summary}

52 sera from three weeks old guinea pigs were studied as a possible source of «subsidiary factor » in the lysis test (= dye test) of toxoplasmosis. 25 per cent of the guinea pigs sera gave interesting results when diluted in Alsever solution of $1 / 10^{\circ}$. The 
same Alsever solution at $1 / 5^{\mathrm{e}}$ allows some improvement to the first results, increasing the activating power of the sera. Without addition of this solution, the non specific hytic power of all the titled sera would induce the total lysis of toxoplasmes. Then it seems that Alsever solution acts both in increasing the activating power and inreducing the non specific hysis of the guinea pig sera.

Le nombre croissant de demandes de séro-diagnostics de toxoplasmose, les difficultés certaines rencontrées dans l'obtention d'un bon complément humain de l'épreuve de lyse nous ont amenés à envisager l'étude des sérums animaux en tant que «facteur accessoire ». Cette étude avait été entreprise, il y a plusieurs années, puisque déjà, en 1954, G. Desmonts (1) rapporte que, selon Sabin et Feldman, les sérums d'animaux, exceptés ceux de la souris et de l'homme, sont inutilisables; ils posséderaient des «anticorps thermolabiles non spécifiques». Dans une communication personnelle G. Desmonts nous rappelle avoir expérimenté, il y a près de 20 ans, le sérum de cobaye, mais « il faut sélectionner les cobayes, en testant chacun d'eux séparément. car beaucoup ont des anticorps spécifiques (toxoplasmose spontanée), et tous ont un fort pouvoir lytique naturel, d'où la nécessité de diluer beaucoup leur sérum ».

En 1967, O. Strannegard (2) parle de l'existence dans les sérums animaux de «facteur hostile au toxoplasme qu'il assimile aux IgA.

En 1968, A. Kobayashi et ses collaborateurs (3), et par la suite G. D. Wallace (4) en 1969, préconisent l'addition de citrate sodique au sérum et même au plasma du «facteur accessoire » améliorant ainsi les résultats de l'épreuve de lyse.

C'est en 1969 que A. Kobayashi et ses collaborateurs (5-6) utilisent avec succès des sérums de cobaye dilués en solution d'Alsever comme complément de l'épreuve de lyse, et c'est à la suite des travaux de cet auteur que nous avons étudié, sur une série de 52 cobayes, les possibilités de «facteur accessoire 》 de ces rongeurs, concernant, d'une part, le pouvoir activateur et, d'autre part, la lyse non spécifique de leurs sérums.

\section{Moyens d'étude.}

- Les animaux utilisés : dans ce travail, nous avons utilisé 52 cobayes de sexe différent, âgés de 6 à 8 semaines environ au moment de la ponction cardiaque. Les sérums recueillis étaient conservés à $-70^{\circ} \mathrm{C}$ jusqu'à leur utilisation.

- Le sérum positif contrôle provenait d'un cas de toxoplasmose lymphadénique humaine positif au $1 / 8000^{\circ}\left(=1200\right.$ U.I.). Nous l'avons utilisé dilué au $1 / 2000^{\circ}$ (= 300 U.I.) pour les différents titrages effectués.

- L'anticoagulant est une solution d'Alsever modifiée suivant la composition :

glucose : $24,5 \mathrm{~g}$;

acide citrique : $8 \mathrm{~g}$;

citrate trisodique : $22 \mathrm{~g}$;

ClNa: $8,5 \mathrm{~g}$;

eau distillée : $1000 \mathrm{ml}$. 
Nous diluons cet anticoagulant au $1 / 5^{\circ}$, au $1 / 10^{\circ}, 1 / 20^{\circ}$ en eau physiologique à $8,5 \%$ et nous l'utilisons pour toutes les dilutions des sérums de cobaye à titrer, du sérum positif contrôle, de même que pour les lavages de l'antigène.

\section{Technique du titrage des sérums de cobaye.}

Nous avons travaillé pour le titrage des sérums de cobayes dans les conditions définies et préconisées par G. Desmonts (7).

L'antigène provient de l'exsudat péritonéal de souris inoculées 2 jours auparavant avec la souche $\mathrm{Rh}$ de toxoplasmes à raison de $0,4 \mathrm{ml}$ d'ascite diluée contenant 15 à 20 parasites par champ, en utilisant un objectif $\times 40$ et des oculaires $\times 8$.

La suspension toxoplasmique est prélevée en totalité, lavée 2 fois en eau physiologique citratée et reconstituée avec $0,3 \mathrm{ml}$ de la même solution d'Alsever puis distribuée à raison d'une goutte dans chaque tube contenant déjà 5 gouttes de sérum de cobaye pur, 5 gouttes de sérum de cobaye dilué au $8 / 10^{\circ}, 5$ gouttes de sérum de cobaye dilué au $6 / 10^{\circ}$. De chacun de ces mélanges, nous reportons 1 goutte dans un tube contenant 1 goutte de sérum positif contrôle dilué au $1 / 2000^{\circ}$ et 1 goutte dans un tube contenant 1 goutte de la solution d'Alsever portée à sa dilution d'emploi. Ces 2 tubes, constituant ainsi les témoins positif et négatif de la réaction, permettent d'étudier le pouvoir activateur et la lyse non spécifique des sérums de cobayes à titrer.

La lecture se fait au microscope à contraste de phase après 30' d'incubation au bain-marie à $37^{\circ} \mathrm{C}$.

Nous avons également titré les sérums de cobaye en eau physiologique à $8,5 \%$ o sans addition d'aucun anticoagulant.

\section{Résultats.}

Envisageons maintenant les résultats de ces différentes expertises qui, malheureusement, étant donné la quantité parfois insuffisante de sérum de cobaye, n'ont pu être toutes menées dans les mêmes conditions.

$1^{\circ}$ En eau physiologique à $8,5 \%$, tous les sérums de cobayes titrés ont présenté une lyse non spécifique dans les témoins négatifs.

$2^{\circ}$ En solution d'Alsever au $1 / 20^{\circ}$, les sérums de cobaye ne possèdent pas de pouvoir activateur suffisant pour permettre la lyse de plus de $80 \%$ des toxoplasmes dans le témoin sérum positif dilué au $1 / 2000^{\circ}$.

$3^{\circ}$ En solution d'Alsever au $1 / 10^{\circ}$, nous obtenons les résultats mentionnés dans le tableau ci-dessous.

Mais, dans ces conditions de dilution de la solution, la moitié seulement des sérums de cobaye peuvent être utilisés dilués au $8 / 10^{\circ}$ ou au $6 / 10^{\circ}$. 
Tableau I. - Nous constatons qu'avec cette dilution de solution d'Alsever, $25 \%$ des sérums de cobayes nous donnent des résultats intéressants, c'est-à-dire moins de $10 \%$ de toxoplasmes lysés dans les témoins négatifs, et plus de $80 \%$ de toxoplasmes lysés dans les témoins positifs

\begin{tabular}{|c|c|c|c|}
\hline $\begin{array}{l}\text { Dilu- } \\
\text { tion du } \\
\text { Sérum de cobaye }\end{array}$ & $\begin{array}{l}\text { Contrôle } \\
\text { négatif }\end{array}$ & $\begin{array}{c}\text { Contrôle } \\
\text { positif }\end{array}$ & $\begin{array}{c}\text { Nombre de } \\
\text { cobayes }\end{array}$ \\
\hline Sérum pur $\ldots \ldots \ldots \ldots \ldots \ldots$ & $\leqslant 10 \%$ & $\geqslant 80 \%$ & 7 \\
\hline Sérum au $8 / 10 \ldots \ldots \ldots \ldots$ & $\leqslant 10 \%$ & $\geqslant 80 \%$ & 6 \\
\hline Sérum au $6 / 10 \ldots \ldots \ldots \ldots$ & $\leqslant 10 \%$ & $\geqslant 80 \%$ & 1 \\
\hline Total $\ldots$. & & & $\begin{array}{l}14 \text { cobayes } \\
\text { sur } 52 \\
\text { soit } 25 \%\end{array}$ \\
\hline
\end{tabular}

Nous avons donc tenté d'améliorer nos résultats en modifiant la dilution de la solution d'Alsever; et nous avons comparé les résultats obtenus avec une dilution au $1 / 5^{\circ}$ de l'Alsever en eau physiologique, aux résultats précédents. Les tableaux ci-dessous permettent de se rendre compte de l'amélioration des résultats, concernant surtout le pouvoir activateur, lorsque la solution d'Alsever est utilisée au $1 / 5^{\circ}$.

Pour la comparaison de ces sérums, nous avons utilisé les sérums de cobayes dilués au $6 / 10^{\circ}$, ce qui nous permet d'avoir un maximum de matériel à exploiter.

Tableau II. - Comparaison des résultats obtenus sur 12 cobayes avec une solution d'Alsever au $1 / 5^{\circ}$ et au $1 / 10^{\circ}$ sur le sérum positif contrôlé au $1 / 2000$.

Nous signalons toujours le pourcentage de Toxoplasmes lysés.

\begin{tabular}{||c|c|c|c|c|c|c|c|c|c|c|c|c||}
\hline $\begin{array}{c}\mathrm{N}^{\circ} \text { des Sérums de } \\
\text { cobayes ........ }\end{array}$ & 16 & 47 & 24 & 49 & 44 & 51 & 50 & 26 & 4 & 42 & 30 & 5 \\
\hline $\begin{array}{r}\text { Solution d'Alsever } \\
\text { au } 1 / 5^{\circ} \ldots \ldots \ldots .\end{array}$ & $98 \%$ & $90 \%$ & $90 \%$ & $87 \%$ & $90 \%$ & $80 \%$ & $70 \%$ & $70 \%$ & $70 \%$ & $99 \%$ & $45 \%$ & $90 \%$ \\
\hline $\begin{array}{c}\text { Solution d'Alsever } \\
\text { au } 1 / 10^{\circ} \ldots . . .\end{array}$ & $9 \%$ & $10 \%$ & $12 \%$ & $9 \%$ & $17 \%$ & $7 \%$ & $7 \%$ & $9 \%$ & $20 \%$ & $50 \%$ & $15 \%$ & $90 \%$ \\
\hline
\end{tabular}

Nous constatons une amélioration très nette du pouvoir activateur des sérums puisque, par exemple, de $9 \%$ de toxoplasmes lysés avec solution d'Alsever au 1/10", nous passons à $98 \%$ quand la même solution est utilisée au $1 / 5^{\circ}$ (cas du sérum $\mathrm{n}^{\circ} 16$ ), ou de $10 \%$ à $90 \%$ (sérums $\mathrm{n}^{\circ}$ 47-24).

Nous avons également comparé les résultats obtenus dans ces 2 cas de dilution de la solution d'Alsever, sur le témoin négatif : 
Tableau III. - Comparaison des résustats obtenus sur 12 sérums de cobayes avec une solution d'Alsever au $1 / 5^{\circ}$ et au $1 / 10^{\circ}$ sur le témoin négatif eau citratée.

Nous indiquons également ici le pourcentage de toxoplames lysés.

\begin{tabular}{|c|c|c|c|c|c|c|c|c|c|c|c|c|}
\hline $\begin{array}{c}\mathrm{N}^{\circ} \text { des Sérums de } \\
\text { cobayes } \ldots \ldots \ldots\end{array}$ & 49 & 44 & 50 & 30 & 16 & 24 & 5 & 42 & 26 & 4 & 47 & 51 \\
\hline $\begin{array}{l}\text { Solution d'Alsever } \\
\text { au } 1 / 5^{\circ} \ldots \ldots \ldots\end{array}$ & $0 \%$ & $2 \%$ & $4 \%$ & $5 \%$ & $7 \%$ & $10 \%$ & $13 \%$ & $15 \%$ & $15 \%$ & $15 \%$ & $25 \%$ & $40 \%$ \\
\hline $\begin{array}{l}\text { Solution d'Alsever } \\
\quad \text { au } 1 / 10^{\circ} \ldots \ldots \ldots\end{array}$ & $8 \%$ & $40 \%$ & $10 \%$ & $30 \%$ & $15 \%$ & $10 \%$ & $8 \%$ & $30 \%$ & $9 \%$ & $4 \%$ & $0 \%$ & $4 \%$ \\
\hline
\end{tabular}

Sur les témoins négatifs, nous obtenons des résultats à peu près similaires dans les deux cas de dilution de la solution d'Alsever.

Nous avons tenté de donner une expression graphique à ces résultats :

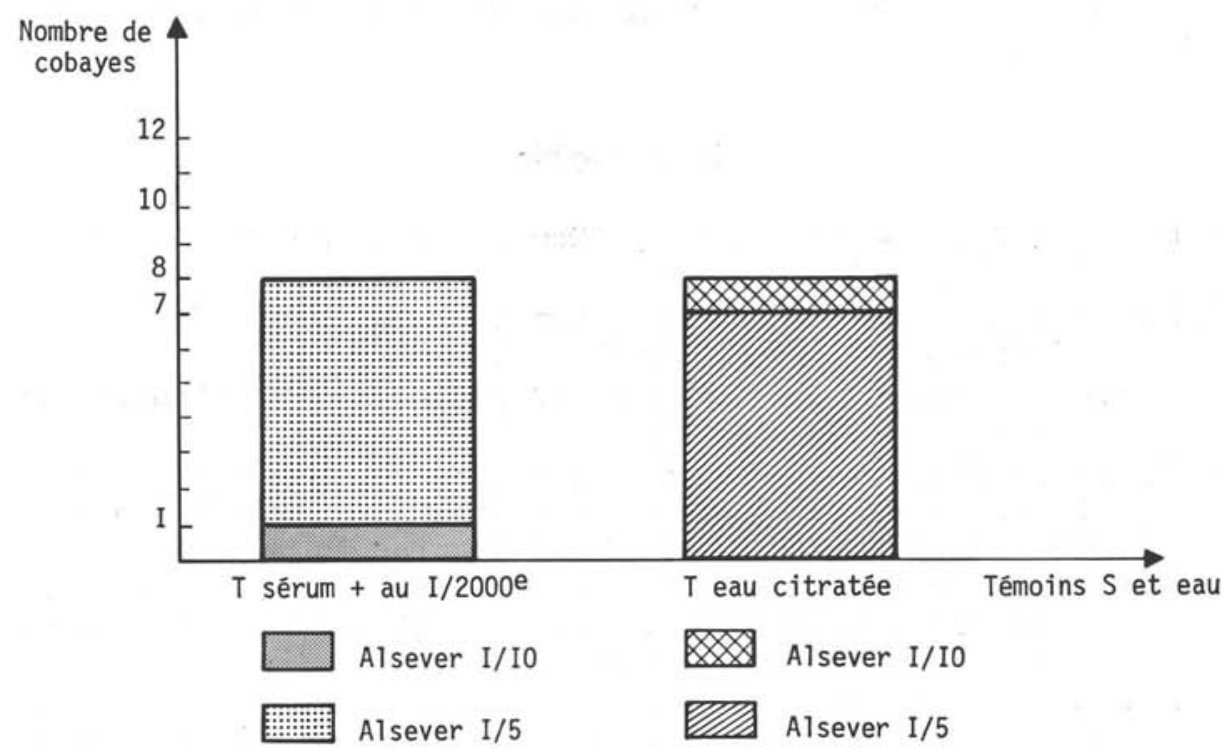

Expression des résultats obtenus avec les 12 sérums de cobayes dilués au $6 / 10^{\circ}$ en solution d'Alsever au $1 / 5^{\circ}$ et au $1 / 10^{\circ}$.

Ce graphique illustre les résultats précédents, où nous observons qu'un seul sérum possède un pouvoir activateur suffisant pour lyser plus de $80 \%$ des toxoplasmes lorsque la solution d'Alsever est diluée au $1 / 10^{\circ}$ alors que 8 sérums ont un pouvoir activateur suffisant lorsque cette solution est diluée au $1 / 5^{\circ}$. 


\section{Conclusion.}

La présence de l'anticoagulant de la solution d'Alsever paraît donc être nécessaire pour l'utilisation du sérum de cobaye en tant que complément de l'épreuve de lyse. La dilution au $1 / 5^{\circ}$ de cette solution en eau physiologique à $8,5 \%$ paraît donner les résultats les plus intéressants pour permettre l'utilisation des sérums de cobayes dilués au $6 / 10^{\circ}$.

L'addition de cette solution d'Alsever à l'eau physiologique semble donc avoir un double effet :

- augmentation du pouvoir activateur des sérums d'une part ;

- diminution de leur lyse non spécifique d'autre part.

Ces premiers travaux nous semblent encourageants pour permettre l'utilisation des sérums de cobayes en tant que complément de l'épreuve de lyse; la quantité de sérum retirée de chaque cobaye étant parfois faible, il paraît nécessaire de pouvoir utiliser ces sérums dilués au maximum, afin d'améliorer la quantité de facteur accessoire utilisable.

\section{Bibliographie}

1. Desmonts (G.), 1954. - Etat actuel du problème de la toxoplasmose humaine. Archives Françaises de Pédiatrie, 11, 157-171.

2. Strannegard (O.), 1967. - The so-called toxoplasma hostile factor and its relation to antibody. Acta Path. Microbiol. Scandinav., 69, 465-476.

3. Kobayashi (A.), Kumada (M.) et Tsunematsu (Y.), 1968. - Effects of anti-coagulants on the dye-test for toxoplasmosis. Japan J. Med. Sci. Biol., 21, 71-89.

4. Wallace (G. D.), 1969. - Sabin-Feldman Dye-test for toxoplasmosis the use of sodium citrate in accessory factor and a method for collecting and storing blood on paper discs. The American Journal of Tropical Medecine and Hygiene, 18, 395-398.

5. Kobayashi (A.), Kumada (M.), Suzuki (M.) et Tsunematsu (Y.), 1969. - Studies on the accessory factor for toxoplasma dye-test. Guinea pig serum as a source of the accessory factor. Japan J. Med. Sci. Biol., 22, 327-336.

6. -,,,,$--- 1970 .-$ Guinea pig serum as a source of the accessory factor for Toxoplasma dye-test. The Journal of Parasitology, 56, Second International Congress of Parasitology, section II, 190.

7. Desmonts (G.) et Cousin (L.), 1963. - Technique de l'épreuve de lyse des Toxoplasmes. Modification du Dye-test de Sabin et Feldman. Feuillets de Biologie, 4, 9-16. 